\title{
Investigation on the design and the stiffness demonstration for a space square-truss deployable mast (STDM)
}

\author{
Hai-jun Luo ${ }^{1,2}$, Qi-feng Cui ${ }^{1,2}$, Ming Li ${ }^{1}$ and Zhi-long Peng ${ }^{1}$ \\ ${ }^{1}$ Aerospace System Engineering Shanghai, Shanghai, 3888 Yuanjiang Road, China \\ ${ }^{2}$ The State Key Laboratory of Mechanical Transmissions, Chongqing University, Chongqing, 174 Shazheng Road, China
}

\begin{abstract}
Space deployable mast is an important device or system, which can deploy and support spacecraft fittings on orbit, such as payload, solar array, antenna, and etc. In order to achieve the pre-function of spacecraft fittings and attitude control of spacecraft, space deployable mast of high stiffness is required. Therefore, it is necessary to analyze the stiffness of the mast to ensure the capability. STDM consisting of longerons, rigid frames, flex-battens, hinge joints, and etc has higher stiffness than coil or articulated deployable mast. The design of STDM is presented, under the demand of future large-scale spacecraft. The theory analysis of stiffness is achieved and the impact factor are found, which establish the theoretical baseline for the optimization design and application of the mechanism product.
\end{abstract}

\section{Introduction}

In recent decades, with the rapid development of space science and technology such as satellite communication, space exploration and earth observation, demands for large spacecraft such as the space station and large satellite is increasing ${ }^{[1-2]}$. As the important component which can change spatial position of spacecraft fittings relative to the mainbody or the overall topology configuration, one-dimensional space deployable mast need to have large size, high stiffness, high load capacity and can deploy and fold repeatedly. The length of STDM is typically greater than $10 \mathrm{~m}$, and the truss should have high stiffness, high positioning accuracy and high reliability. Currently, the mast has been successfully applied in the International Space Station to expand and support large-area flexible solar array ${ }^{[3-6]}$.

Fundamental frequency and stiffness property of payload on orbit is directly determined by the stiffness characteristic of expanding space square truss mast ${ }^{[7]}$. Flex-battens, longerons and diagonal cables are key components of the truss, whose performance directly affects the stiffness property of mast. Therefore, investigating the stiffness characteristic of the truss in the initial stages of design, the axial stiffness, bending stiffness and torsional stiffness should be quantified, so that the key factors affecting the truss stiffness property can be identified in order to provide an effective baseline for optimization design of the following products.

\section{Structural design and working principle}

\subsection{Structural design}

STDM is mainly composed of deployable truss, collection system and drive-transmission system. The deployable state of mast is illustrated in Figure 1.

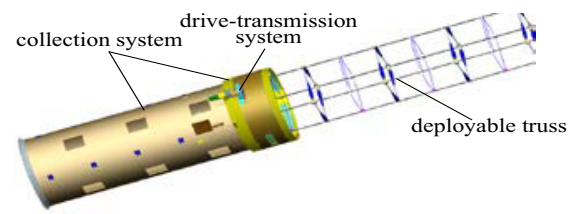

Figure 1. Compositions of STDM.

The truss is a square variable topology which can extend and retract repeatedly, mainly assembled by longerons, rigid frames, elbows, flex-battens, hinge joints, diagonal cables and etc, as illustrated in Figure.2. It's the main load-bearing mechanism of mast. Four flex-battens being post-buckling consist of a flexible frame, whose configuration transform between bend and stretch process lead to rectilinear motion of rigid frame, that's why the truss can deploy and retract. Relative to trilateral-shaped structure, quadrilateral structure has greater moment of inertia and bending stiffness, better mechanical property, which leads to a stable configuration.

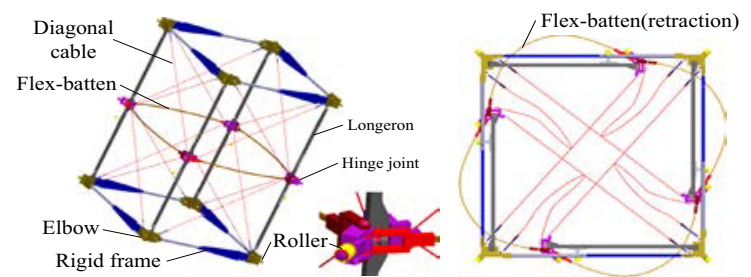

Figure 2. The truss being extended and retractable of STDM. 
As illustrated in Figure.3, rigid frame can be seen as a rigid plane whose square shape remains invariant during the extension/retraction operations. In addition, the upper and lower longerons hinge with rigid frame and flexible frame, and then four nodes of flexible frame form a quadrangle whose side length increases or decreases during the extension/retraction operations of mast.

It should be noted that the outer envelope radius of flex-batten after deformation should not be greater than the radius of the truss $R$ during the extension/retraction operations of mast. Thereby, kinematical trajectory of rigid frame $A_{2} B_{2} C_{2} D_{2}$ and flexible frame $A_{3} B_{3} C_{3} D_{3}$ is limited within a cylinder whose bottom is the circumscribed circle of four nodes of rigid frame $A_{1} B_{1} C_{1} D_{1}$, and the track of each node of $A_{2} B_{2} C_{2} D_{2}$ is a straight line in the axial direction of the cylindrical surface, while the track of each node of $A_{3} B_{3} C_{3} D_{3}$ is a spatial rotation curve whose trajectory radius $r$ varies.

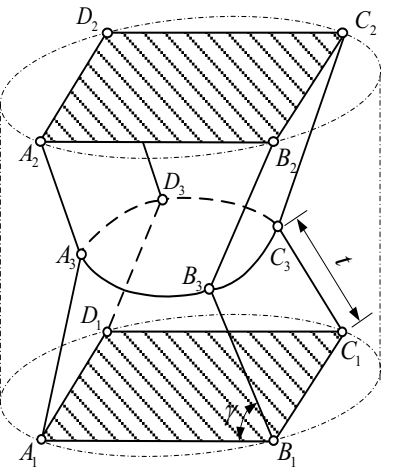

(a)

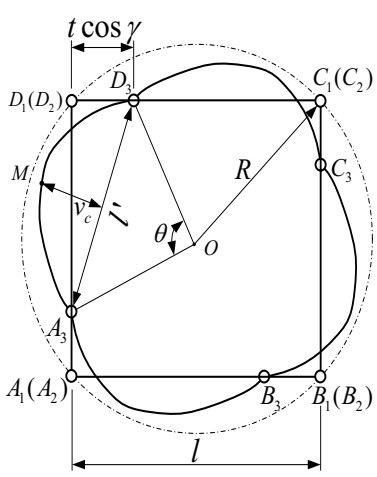

(b)
Figure 3. Analysis of deployment-retract kinematic principle of flexible frame.

As shown in Figure.3(b), the relation between $D_{1} D_{3}$, $A_{3} D_{1}, A_{3} D_{3}$ can be expressed as,

$$
{\overline{D_{1} D_{3}}}^{2}+{\overline{A_{3} D_{1}}}^{2}={\overline{A_{3} D_{3}}}^{2}
$$

Where $D_{1} D_{3}$ and $A_{3} D_{1}$ is respectively the distance of corresponding or adjacent hinge joints between flexible frame and rigid frame within the flexible frame plane, and then it can be deduced that $\overline{D_{1} D_{3}}=t \cos \gamma$ and $\overline{A_{3} D_{1}}=l-\overline{D_{1} D_{3}}=l-t \cos \gamma, A_{3} D_{3}$ stands for the distance of adjacent hinge joints or the span across the post-buckling flex-batten after bended,

$$
\begin{aligned}
& l^{\prime}=\sqrt{(t \cos \gamma)^{2}+(l-t \cos \gamma)^{2}} \\
&=t \sqrt{(\sqrt{2} \cos \gamma-R / t)^{2}+(R / t)^{2}} \\
& \text { the span between upper and lower fram }
\end{aligned}
$$

Where $t$ is the span between upper and lower frames or the length of longerons, $R$ is the outer envelope radius of the truss, $l=\sqrt{2} R$ is the side length of rigid frame or the span across the flex-battens when the truss bay is expanded state, $\gamma$ stands for the included angle of longerons and hinged rigid frames during the extension/retraction operations of bay.

\subsection{Working principle}

With elastic bending deformation of flex-batten and the pin joint between upper and lower longerons, the truss can only completely stow; with rotation of drive nut and release of elastic strain energy that flex-batten stored, the truss can only achieve the overall extension.

Figure. 4 shows how the upper and lower longerons can be treated as the legs of a card table that fold in perpendicular directions to each other. Figure. 5 shows flex-battens movement during the extension/retraction operations of the truss.
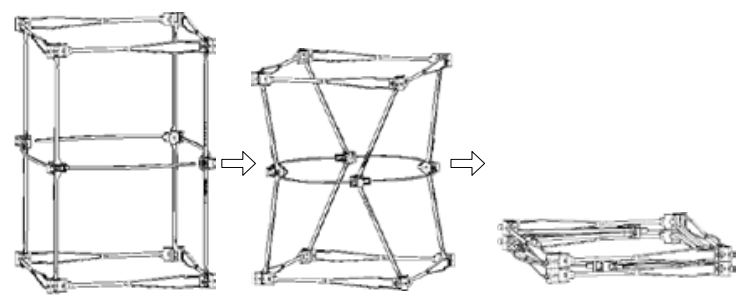

Figure 4. Longerons movement.

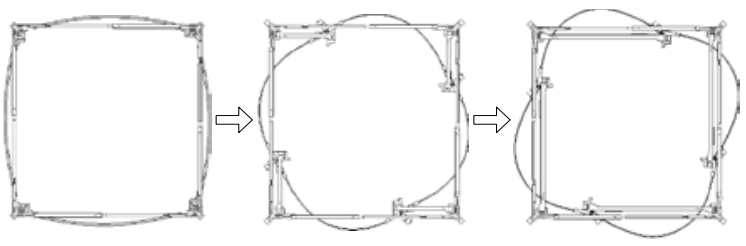

Figure 5. Flex-battens movement.

The truss is a mechanism consisting of numbers of bays, the component diagram which shows how the mast deploys and retracts is illustrated in Figure. 6 according to the structure and principle.

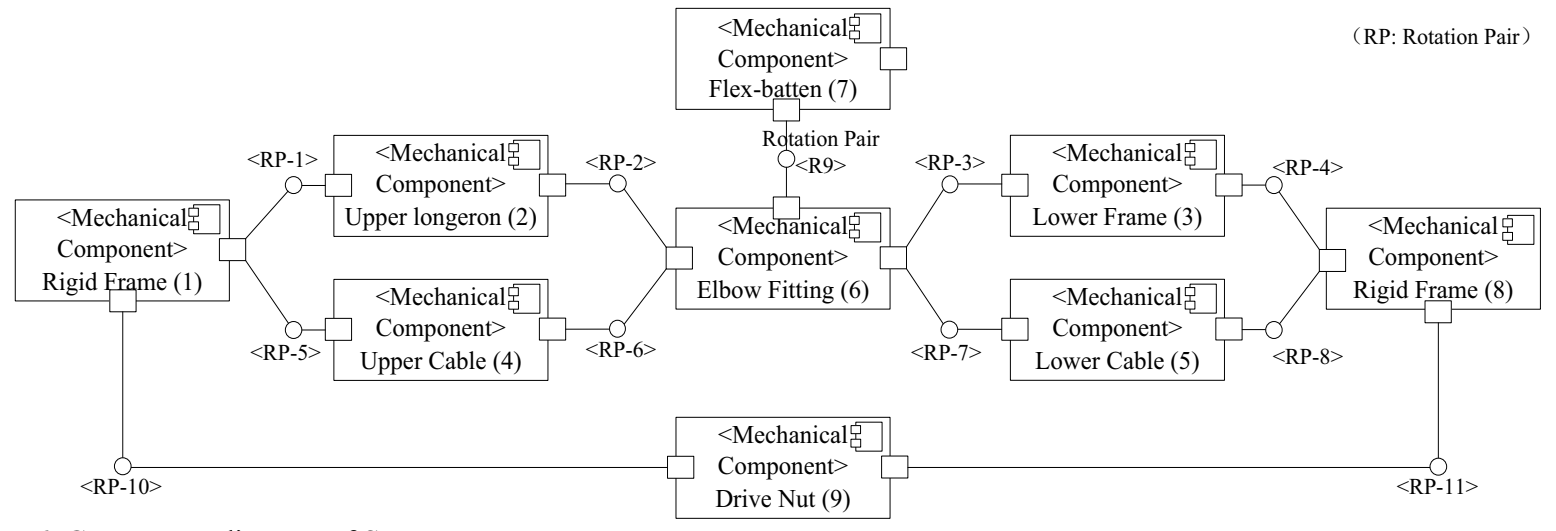

Figure 6. Component diagram of STDM. 

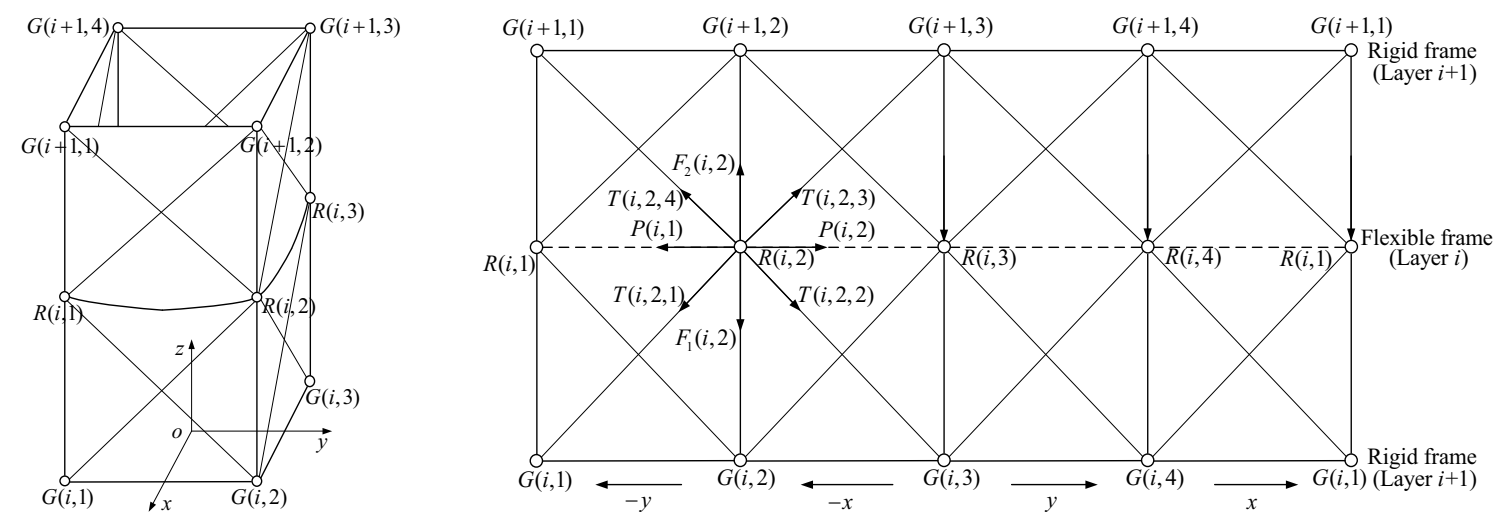

Figure 7. Mechanical state diagram of the truss bay.

\section{Stiffness analysis of the truss}

The truss is the main load-bearing parts of mast, by investigating the stiffness characteristic of the truss, the axial stiffness, the bending stiffness and the torsional stiffness can be quantified, furthermore, the key factors affecting the truss stiffness property can be identified. It is worth noting that the preload of the flex-batten is provided by the diagonal cable in truss bay. There are some assumptions following in order to simplify the stiffness analysis appropriately:

(1) Diagonal cables are regarded as the linear material, the initial preload of each cable is equal and the preload would not be reduced to zero under slightly deformation state for the truss. Then the truss can be considered with linear mechanical characteristics;

(2) Flex-batten will not further post-buckle when the truss is being deployed;

(3) The connecting gap of elbow or hinge joints are ignored.

Figure.7 illustrates the mechanical state of one of the truss bays, $O$ stands for origin of fixed coordinate system and it's geometric centre of the rigid frame named layer $i$, there is three translational degrees of freedom at each node. Among others, $i=1,2, \cdots N$ is the layer number of rigid/flexible frame, $j=1, \cdots 4$ is the node number of four quadrants corresponding layer $i, k=1, \cdots 4$ is four diagonal cables number corresponding layer $i$ and node $j . G(i, j) 、 R(i, j)$ are nodes of rigid/flexible frame respectively, $F_{1}(i, j) 、 F_{2}(i, j)$ are elastic internal force of upper and lower longerons respectively, $P(i, j)$ is the internal tension of flex-batten, $T(i, j, k)$ is the tension of diagonal cables being in rigid/flexible frame nodes.

\subsection{Analysis of the axial stiffness}

The cross section of the truss is quadrangle and it is intuitional that when an axial force is applied on the truss, the force of each node can be treated as $F / 4$.

\subsubsection{Analysis of the tension stiffness}

Suppose that $F$ is a tensile force, Figure. 8 illustrates the axial mechanical analysis of longeron at node $R(i, 2)$, $R^{\prime}(i, 2)$ stands for the position of $R(i, 2)$ after the longeron is deformed.

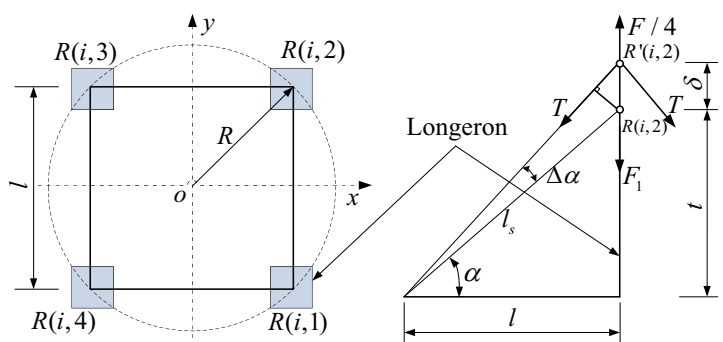

Figure 8. The mechanical analysis of cross section and one node of the truss bay.

As the above illustration shows that,

$$
\begin{aligned}
\frac{F}{4} & =2 T \sin (\alpha+\Delta \alpha)+F_{1} \\
& =\frac{2 E_{s} A_{s}}{l_{s}} \delta \sin ^{2}(\alpha+\Delta \alpha)+\frac{E A}{t} \delta
\end{aligned}
$$

Herein, $l_{s}=\sqrt{l^{2}+t^{2}}=\sqrt{2 R^{2}+t^{2}}$,

$$
F=\frac{8 E_{s} A_{s}}{\sqrt{2 R^{2}+t^{2}}} \delta \sin ^{2}(\alpha+\Delta \alpha)+\frac{4 E A}{t}
$$

In the above equation, $E_{s} 、 A_{s}$ stands for elasticity modulus and cross-sectional area of the cables respectively, $E 、 A$ and $t$ stands for elasticity modulus, cross-sectional area and length of the longeron respectively, $R$ is the outer envelope radius of the four longerons, $l=\sqrt{2} R$ is the span between longerons or side length of frames and there is $l=\sqrt{2} R, \delta$ is the tensile deformation of longerons in axial direction. It can be deduced $\sin \alpha=t / l_{s}$, therefore, the tension stiffness can be obtained,

$$
\begin{aligned}
{[E A]_{2}=\lim _{\Delta \alpha \rightarrow 0} \frac{F t}{\delta} } & =\lim _{\Delta \alpha \rightarrow 0}\left(\frac{8 E_{s} A_{s} t}{\sqrt{2 R^{2}+t^{2}}} \sin ^{2}(\alpha+\Delta \alpha)+4 E A\right) \\
& =8 E_{s} A_{s}\left[2(R / t)^{2}+1\right]^{-\frac{3}{2}}+4 E A
\end{aligned}
$$


The side length of the cross section of longeron is $p$ and its modulus $E=70 \mathrm{Gpa}$, the diameter of cables is $d$ and its modulus $E_{s}=60 \mathrm{Gpa}$. The curve varying from $R / t$ of the tension stiffness of the truss is illustrated in Figure 9 . It can be concluded that, with the increase of $R / t$ the stiffness shows a non-linear decreasing trend; the change of the cable diameter hardly affects the stiffness; while increasing the cross-sectional dimension of longeron can enhance the stiffness significantly.

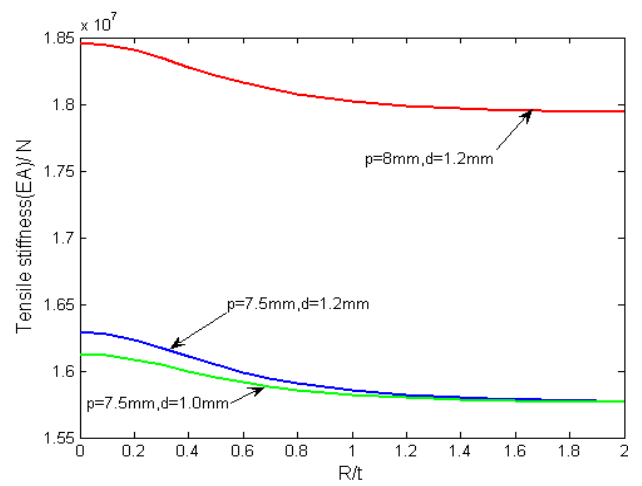

Figure 9. Tension stiffness curve of the truss.

The conclusion that the cable stiffness contribution to tension stiffness is negligible through the analysis and the calculation. In summary, the tension stiffness of the truss can be obtained,

$$
[E A]=4 E A
$$

\subsubsection{Analysis of the compression stiffness}

Suppose that $F$ is a compressive force, the cable has no contribution to the compression stiffness of the truss for the cable cannot withstand the pressure. Then the compression stiffness is,

$$
[E A]^{\prime}=4 E A
$$

It shows that increasing the cross-sectional dimension of longeron can enhance the stiffness significantly.

\subsection{Analysis of the bending stiffness}

The stiffness contribution of cables to the bending stiffness of the truss is negligible when ignoring the rotation gap of elbow or hinge joints. Figure. 10 shows the state that the truss bay is placed at any angle.

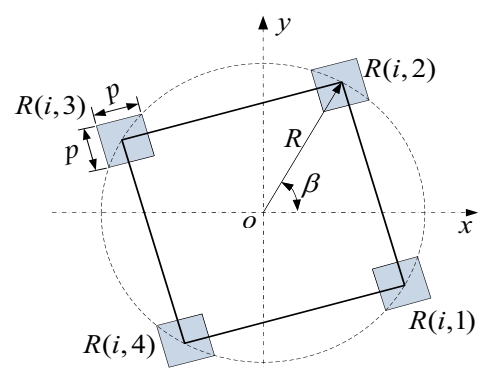

Figure 10. Schematic diagram of the bay placed at any angle.
According to the translation theorem and rotationaxis theorem, the moment of inertia of $x$ axis is,

$$
\begin{aligned}
I_{x} & =4 \times \frac{p^{4}}{12}+p^{2}(R \sin \beta)^{2}+p^{2}\left[R \sin \left(\beta+\frac{\pi}{2}\right)\right]^{2}+ \\
& p^{2}[R \sin (\beta+\pi)]^{2}+p^{2}\left[R \sin \left(\beta-\frac{\pi}{2}\right)\right]^{2} \\
& =\frac{p^{4}}{3}+2 p^{2} R^{2}
\end{aligned}
$$

Herein, $\beta$ is the angle of the cross-section of the truss and axis $x$. It can be concluded from the equation that the moment of inertial is independent with $\beta$. In addition, the moment of inertia of longeron itself is negligible when $p \ll R$. Then is the bending stiffness of the truss,

$$
E I=2 E A R^{2}
$$

Figure.11 illustrates the quadratic curve changed with the outer envelope radius $R$. From the curve, it can be summarized that the change of the cross-sectional dimension of longeron has little effect on the bending stiffness of the truss when $R$ is small enough. Obviously, when $R$ increases to a certain size, such as $R \geq 200 \mathrm{~mm}$, the bending stiffness becomes more sensitive to changes in cross-sectional dimension. Also, the material of longeron is an important factor affecting bending stiffness of the truss, which means longerons with high elastic modulus can significantly improve the bending stiffness. For example, the bending stiffness of the truss will increase about $57 \%$ if the aluminium alloy is replaced by titanium alloy for longeron when $R=300 \mathrm{~mm}$.

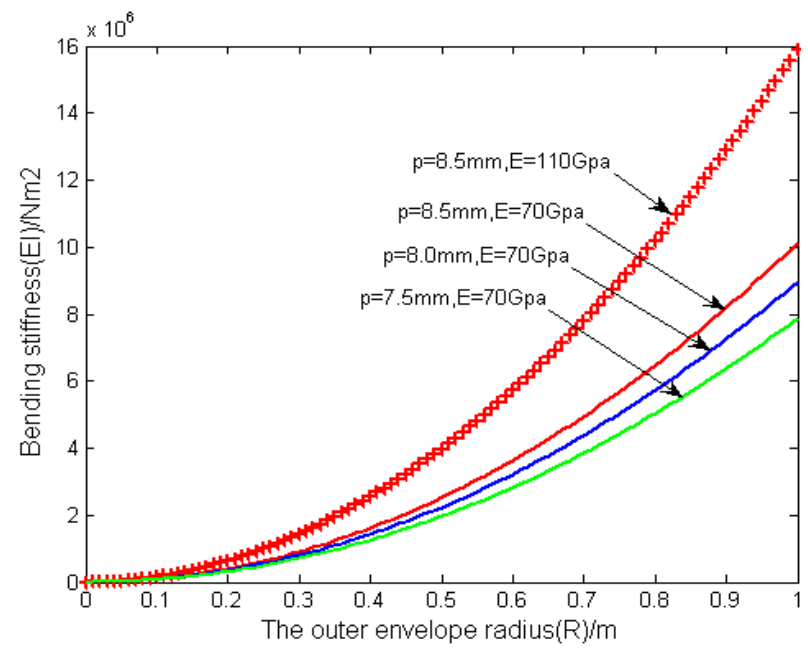

Figure 11. Bending stiffness curve of the truss.

\subsection{Analysis of the torsional stiffness}

The upper and lower frames will result in torsional deformation when the truss bears a torque $T^{\prime}$, as illustrated in Figure 12. 


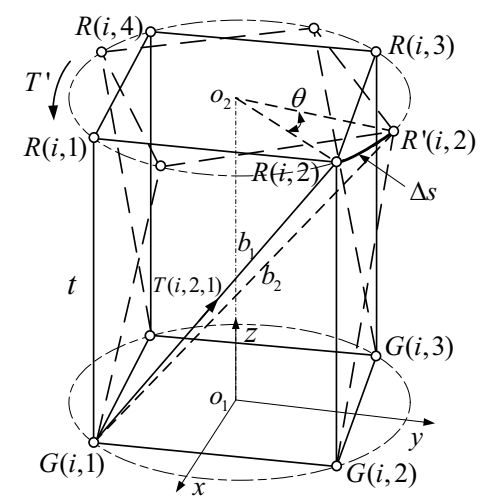

Figure 12. Schematic diagram of torsional deformation adjacent frames of the truss bay.

The truss bay results in small-angle $\theta$ torsion because of $T^{\prime}$, internal force of left diagonal cable becomes smaller and the right diagonal cable tightens at the same time the length extend to $b_{2}$ from initial length $b_{1}$, then the horizontal displacement in the $y z$ plane of point $R(i, 2)$. Small angle $\theta$ approximately is,

$$
\Delta l \approx \Delta s=R \theta
$$

The calculation model of the tensile deformation of cable is expressed in Figure.13.

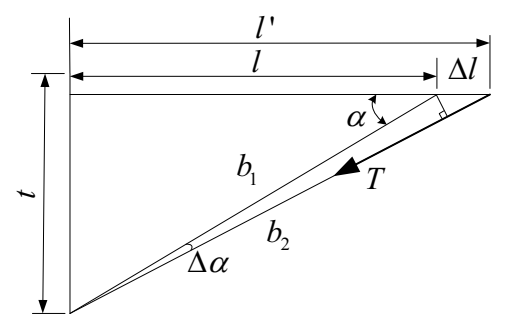

Figure 13. Calculation model of tensile deformation of cable.

The tensile force can be calculated from the torsion,

$$
\begin{aligned}
T=\frac{E_{s} A_{s}}{b_{1}}\left(b_{2}-b_{1}\right) & =\frac{E_{s} A_{s}}{t} \sin \alpha \Delta l \cos (\alpha-\Delta \alpha) \\
& =\frac{E_{s} A_{s} R \theta}{t} \sin \alpha \cos (\alpha-\Delta \alpha)
\end{aligned}
$$

Considering that when the truss bears respectively torque load whose direction is positive or negative, the torsional stiffness contribution of longeron to the truss is zero because the longeron has rotational degrees of freedom in the direction of the truss stowing. Based on the analysis, the truss suffers a torque $T^{\prime}=4 T R \cos \alpha$, then the torsional stiffness of the truss,

$$
\begin{aligned}
G I_{p} & =\lim _{\Delta \alpha \rightarrow 0} \frac{T^{\prime} t}{\theta} \\
& =\lim _{\Delta \alpha \rightarrow 0} 4 E_{s} A_{s} R^{2} \sin \alpha \cos \alpha \cos (\alpha-\Delta \alpha) \\
& =4 E_{s} A_{s} R^{2}\left(1+\frac{1}{2(R / t)^{2}}\right)^{-1}\left(1+2(R / t)^{2}\right)^{-\frac{1}{2}}
\end{aligned}
$$

Figure.14 illustrates the torsional stiffness curve of the truss changed with $R / t$. The curve shows that $R$ has little effect on the torsional stiffness while diameter of the cable is the a key factor. Obviously, increasing the diameter of the cable is an effective way to improve the torsional stiffness, and with $R / t$ increases, the torsional stiffness increases when $0<R / t<1$ and decreases when $R / t>1$.

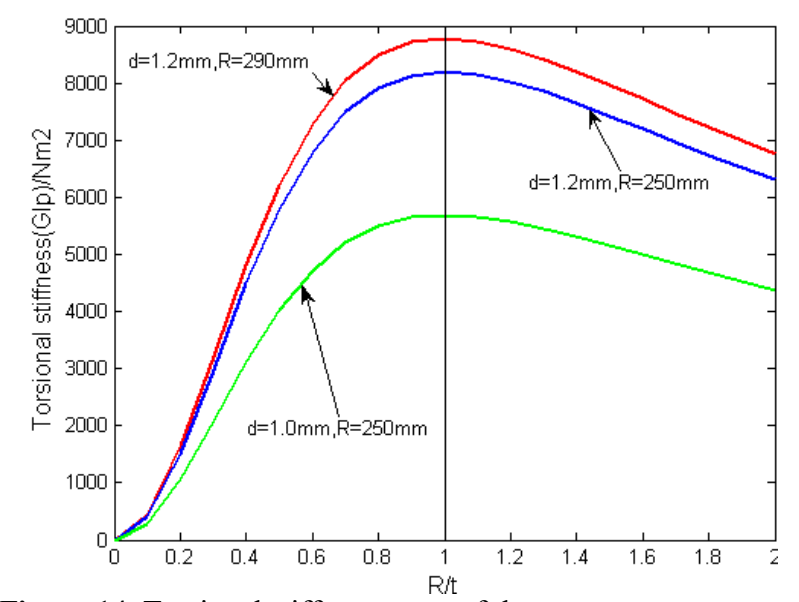

Figure 14. Torsional stiffness curve of the truss.

When $R / t=1$,

$$
\left[G I_{p}\right]_{\max }=\frac{8 E_{s} A_{s} R^{2}}{3 \sqrt{3}}
$$

\subsection{Discussion of the stiffness analysis}

The aforementioned analyses of stiffness characteristic of mast are carried out under some assumptions, while the analysis can help identifying several key factors affecting stiffness. Under the conditions that the materials are certain and the weights meet the requirements of mast, the diameter of cable, the cross-sectional area of longeron and the ratio $R / t$ all are important factors. In the engineering design, it needs to consider the overall mutual influence relations between the axial stiffness, the bending stiffness and the torsional stiffness so that the stiffness characteristics of mast can be optimized.

Comprehensive consideration of the conditions on orbit, when there is $R / t=1$ that the stiffness of the truss is more appropriate, and then the whole structure of mast will be in a good coordination. Figure.15 shows structures of the quadrilateral and trilateral configuration and the stiffness comparison between them shows in Table 1.

Table 1. Stiffness comparison between the square truss and trilateral configuration structure ${ }^{\mathrm{a}}$.

\begin{tabular}{|c|c|c|c|}
\hline $\begin{array}{c}\text { Stiffness } \\
\text { of truss }\end{array}$ & $\begin{array}{c}\text { Quadrilateral } \\
\text { configuration }\end{array}$ & $\begin{array}{c}\text { Trilateral } \\
\text { configuration }\end{array}$ & Ratio \\
\hline $\begin{array}{c}\text { Axial } \\
\text { stiffness }\end{array}$ & $4 E A$ & $3 E A$ & $133.33 \%$ \\
\hline $\begin{array}{c}\text { Bending } \\
\text { stiffness }\end{array}$ & $2 E A R^{2}$ & $\frac{3}{2} E A R^{2}$ & $133.33 \%$ \\
\hline $\begin{array}{c}\text { Torsional } \\
\text { stiffness }\end{array}$ & $\frac{8 E_{s} A_{s} R^{2}}{3 \sqrt{3}}$ & $\frac{2 E_{s} A_{s} R^{2}}{\sqrt{3}}$ & $133.33 \%$ \\
\hline
\end{tabular}

\footnotetext{
${ }^{a}$.Design parameters of the truss: $R=t$
} 

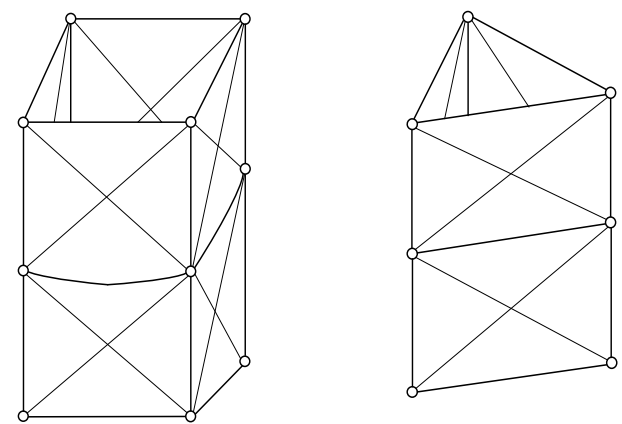

Figure 15. Structures of the quadrilateral and trilateral configuration.

Comparing with the trilateral configuration structure, the quadrilateral configuration has obvious advantages about stiffness under the same basic dimensions of the truss. In general, increasing the number of longerons can effectively improve the deployable stiffness of truss but weakening influence of weight of mechanism.

\section{Conclusion}

Large one-dimensional space deployable mechanism is widely used in space science presently, commonly used for long-distance extension and high-precision positioning of space payload. The working principle of the STDM is based on the extension and retraction of the truss so that the mast can deploy or fold. Obviously, the extension stiffness of the truss decides the fundamental frequency and work performance on orbit of spacecraft fittings. Firstly this paper proposed a design scheme of a quadrilateral mechanism, and analyzed the relationship of components during the extension/retraction operations of the truss. Then the paper analyzed the axial stiffness, the bending stiffness and the torsional stiffness with analytical method by simplifying certain models, and pointed out several effective measures enhancing the stiffness.
Parametric analysis of the stiffness can provide a valid basis in favor of structure optimization, performance improvement, and risk reduction of product development. Stiffness testing of principle prototype will be carried out in the following work to verify the analyses above.

\section{Acknowledgement}

The authors are grateful for the supports received from the open program from the state key laboratory of mechanical transmissions, Chongqing University (SKLMT-KFKT-201404).

\section{References}

1. Cui Qifeng, Peng Zhilong. Study on the design and stiffness for a space articulated deployable mast[C]. 3RD National Manned Space Congress, 1-2 (2014) (in Chinese)

2. Shen Bin. Design and Characteristic Analysis of the Coilable Lattice Mast[D]. Shanghai: Shanghai Academy of Spaceflight Technology, 1-3 (2007) (in Chinese)

3. Elliott, Kenny B. Model Calibration Efforts for the International Space Station's Solar Array Mast[R]. AIAA-NF1676L-13312, 1-3 (2012).

4. Knight, Norman F, Jr. FAST Mast Structural Response to Axial Loading: Modeling and Verification[R]. AIAA-NF1676L-13313, 1-2 (2012).

5. Karen M.B. Taminger. Solid Freeform Fabrication: an Enabling Technology for Future Space Missions. Keynote Lecture for 2002 International Conference, San Antonio, 4 (2002)

6. Bowden M, Benton M. Design of deployable-truss masts for space station [C]// AIAA/AHS/ASEE Aerospace Design Conference, Goleta ca, 1-10 (1993)

7. Deng Zongquan. Design of Space Deployable and Foldable Mechanisms[M], 10-12 (2013) (in Chinese). 